\title{
Modeling continuous shape change for facial animation
}

\author{
JULIAN J. FARAWAY \\ Department of Statistics, University of Michigan, Ann Arbor, MI 48109, USA \\ faraway@umich.edu
}

Received May 2003 and accepted May 2004

\begin{abstract}
The movement of landmarks on the human face can be recorded in 3D using motion capture equipment. We describe methods for the analysis of data collected on groups of subjects with a view to describing and assessing the differences between the facial motions of those groups. We focus on the smile motion in particular. The methods presented can be used more generally for continuous shape change data.

We introduce a novel parameterization of shape change that allows the parsimonious description of facial motion. We allow for a distinction between static facial shape and dynamic facial motion. We describe statistical methods for modeling differences in facial motion including a comparison of mean motions, principal components for describing the variation in motion and linear models for describing the effects of predictors.
\end{abstract}

Keywords: functional data analysis, shape data, registration, B-splines, multidimensional scaling

\section{Introduction}

Surgical procedures for repairing and correcting facial injuries and defects have advanced considerably in recent years. Surgeons are able to make impressive improvements to facial appearance. However, more than just a pretty face is needed - the face must function well too. Eating, speaking and facial gestures are an essential part of life. Function, as well as form, must be considered when evaluating surgical outcomes.

Cleft lip or palate is a relatively common birth defect. The standard treatment involves a series of surgeries through childhood to correct the problem. The incremental improvements in facial form tend to decrease with each successive surgery while increasing the risk of scarring and nerve damage that can degrade facial function. Decisions need to be made by the surgeon and parents about whether to continue. Aesthetic considerations tend to have greater weight than they perhaps deserve and small improvements in form may be obtained at the cost of function. The full quantification of facial function that would allow for more informed decisions has not been available. In earlier work-(Trotman, Faraway and Essick 2000), we proposed some measures but the ones presented here represent a full step beyond to represent the full dynamic motion of the face.
Another type of surgery where additional understanding of facial function is needed involves persons with unusually small (retrognathic) or large (prognathic) jaws. Surgical techniques have been developed to modify jaw size closer to the norm but less is understood about how the movement of persons with retrognathic or prognathic jaws differs from the the norm and how surgery affects this movement. The data we analyze below is drawn from a study investigating these issues.

The methods we shall present below are useful for quantizing and modeling facial motion for clinical purposes. They also have some application to non-clinical applications in animation and in modeling shape change in general.

Three dimensional motion cannot be satisfactorily represented statically on a page. To appreciate our motion models, they must be seen in action. We have developed standalone viewer software that must be seen to understand the outcomes of our analyses.

\section{Data}

The sample analyzed below consisted of 48 healthy subjects recruited from the University of North Carolina School of Dentistry Orthodontic and Dentofacial Clinics. Some of the subjects had retrognathic and prognathic jaws. The extent of these 
variations in facial form can be quantified in various ways. We will use a measure called MFLF which represents the ratio of a midface distance (nasion to anterior nasal spine) to a lower face distance (anterior nasal spine to menton). There are other measures that would be considered in a full analysis including age and gender, but we aim to present only the method of analysis here. Subjects were asked to perform various standard facial motions - eye open, eye close, cheek puff, lip purse, grimace and smile. We shall focus on the smile only here although a full analysis would study the other animations. Subjects were instructed to bite on the back teeth and smile as much as possible. This is artificial but a previous study indicated that this smile had similar characteristics to natural smiles - see Mendez (1999). Each animation was repeated 3 times.

Data may be collected on facial motion using motion capture equipment. Twenty four retro-reflective markers, each with a diameter of $2 \mathrm{~mm}$ were attached using eyelash adhesive to specific sites on the face shown in Fig. 1.

Some markers can be placed quite consistently. For example, the position of the commissures (lip corners-markers 16 and $19)$ is well defined. Other markers are placed on landmarks that are less well defined-for example, markers 13 and 14 on the upper lip. This is important in our choice of measures in the data analysis to follow.

The Motion Analysis video tracking system used four cameras to track the motion at a rate of 60 frames per second. All the motions were recorded for 3 seconds for a total of 180 frames per motion. At least two cameras must have a line of sight to a given marker for its position in three dimensions to be recorded. Due to the redundancy of having four cameras, missing data was relatively rare although occasional outliers are generated

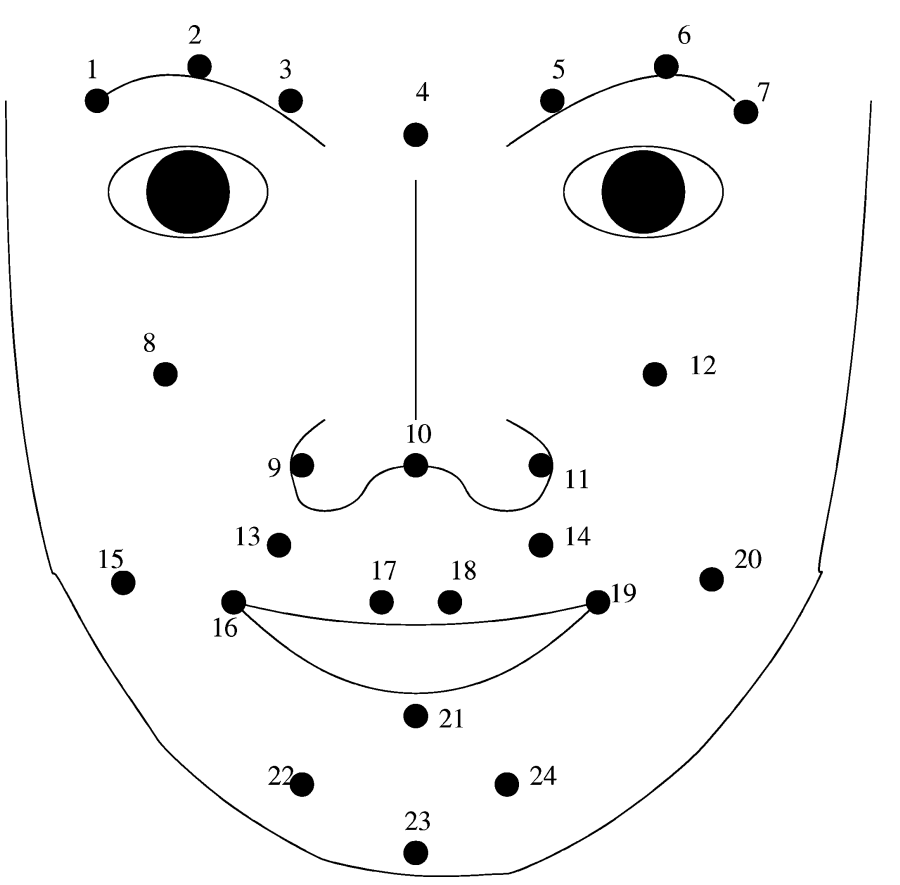

Fig. 1. Schematic of facial marker placement due to problems with tracking the data were encountered. Note that the head was not constrained so that the motion of face was confounded with motion of the whole head. Each motion is represented by $24 \times 3 \times 180=12960$ numbers and there were 144 smiles observed for a total of around 1.9 million numbers in the complete dataset.

We show two smiles by the same person in Exhibit 1. (Please see Appendix for description of how to obtain and operate the viewing software. It is important to view the motion from the side as well as the front). In just these two observations, we see several important characteristics of the data. The head is not constrained. The initial positions are not exactly (nor could they be) aligned. The smiles take place at different times during the 3 second period. A small amount of noise due to measurement error is visible.

We have presented only a representative selection of analyses of this data. Our purpose is just to describe what is possible in a concrete manner but without attempting a comprehensive analysis of the data.

\section{Methods}

\subsection{Shape}

The configuration of the 24 markers constitute a shape (see Dryden and Mardia (1998), Bookstein (1991) and Small (1996) for introductions to statistical shape analysis). However, we are not so much interested in the shape of the face itself, but rather in how this shape changes when the face moves. We already know that facial shapes differ from person to person and what distinguishes those with retrognathic jaws from those with the normal jaws. Our interest is in the motion itself independent of the shape - essentially the derivative of the shape as it changes over time. This explains the necessity of developing new techniques.

We choose to base our analysis on the following type of measure: Let $d_{i j}(t)$ be the (Euclidean) distance between marker $i$ and $j$ at time $t$, where $i, j=1, \ldots n$ and $n$ is the number of markers. Now let

$$
r_{i j}(t)=\frac{d_{i j}(t)}{d_{i j}(0)}-1,
$$

which represents the relative change in the distance from rest. This measure has several desirable properties:

1. It is invariant to whole head motion.

2. It is approximately invariant to small variations in marker placement. As we mentioned above, the landmarks for several of the markers are not precisely defined. Because of the relative scaling, small variations in placement will have only have a second order effect.

3. It is not dependent on local shape. For example, consider the distance between the commissures, 16-19. Of course, some people have bigger mouths than others but we have little interest in this. We are more concerned with how this 
distance changes during, say, a smile. By scaling using the initial distance, we remove much of the this effect.

In Lele and Richtsmeier (2000) and other research articles, an approach to statistical shape analysis based on $d_{i j}$ over all pairs of distances called EDMA (Euclidean Distance Matrix Analysis) is developed. Given the matrix $d_{i j}$, it is possible to reconstruct the shape (up to rotation, translation and reflection).

\subsection{Reconstruction}

Suppose we were to average (elementwise) two $d$ matrices representing different faces. $\bar{d}$ would almost certainly not correspond exactly to any configuration of points in 3D. However, provided the two shapes are not very different, we expect that a face exists that has a distance matrix with entries close to $\bar{d}$. This is the classical multidimensional scaling (MDS) problem for which a standard textbook solution exists.

A further difficulty is that MDS does not fix the orientation of the face. We will require that successive frames in the reconstruction as $t$ varies be displayed continuously. There are several solutions to this. In our case, we identify 4 markers on the nose, 4, 9, 10 and 11, that do not move very much relative to each other. We align successive frames so that the nose appears upright. In other applications, particularly where the shape lacks a fixed feature, like the nose in this example, alignment is more problematic and other schemes would need to be considered. For example, Procrustes fitting could also be used to ensure smoothness between frames.

\subsection{Registration}

The subjects are instructed to start from a neutral facial pose, assume the required pose, such as "smile", and then relax. This should be completed within 3 seconds starting from a cue to the subject that the cameras are recording. Subjects are given the opportunity to practice and if they miss the 3 second time slot, they have the opportunity to repeat the animation.

There are five phases to the motion:

1. Neutral pose

2. Assume the required pose

3. Hold

4. Relax from the required pose

5. Neutral pose

This means there are 4 transition times. These times will vary from animation to animation so it would not make sense simply to average several motions cross-sectionally in time. Motions need to be "registered" with each other so that comparable points in the motion are averaged. A further difficulty is that it is difficult to precisely identify these transition times.

We illustrate the issues in Fig. 2, where information from a smile is depicted. The first panel shows a Lowess-smoothed $r_{13,17}(t)$ which represents a distance on the upper lip. The five phases of the motion are clearly identifiable although the precise transitions are not unambiguous. The center panel shows $r_{4,5}(t)$ which represents a distance above the eye. In this case the phases are not identifiable, not perhaps unsurprisingly as movement during a smile is confined to the lower part of the face while other unrelated activity may take place above the eyes. Clearly, we'd prefer to use the first plot to choose the transitions in this example. Unfortunately, patterns will differ from animation to animation and from individual to individual. What may be a good pair for one motion might not be for another. For this reason, we compute the average $r_{i j}(t)$ over all pairwise distances as shown in the right panel and use this to select the transitions.

Various methods for automatically registering curves have been developed - see for example Ramsay and Li (1998). The drawback of automatic methods in our experience is that, although good ones may work well enough most of the time, they can fail badly when confronted with unexpected features. Given the outliers and missing values in the data, such anomalous cases are not uncommon. For this reason, we manually identify the
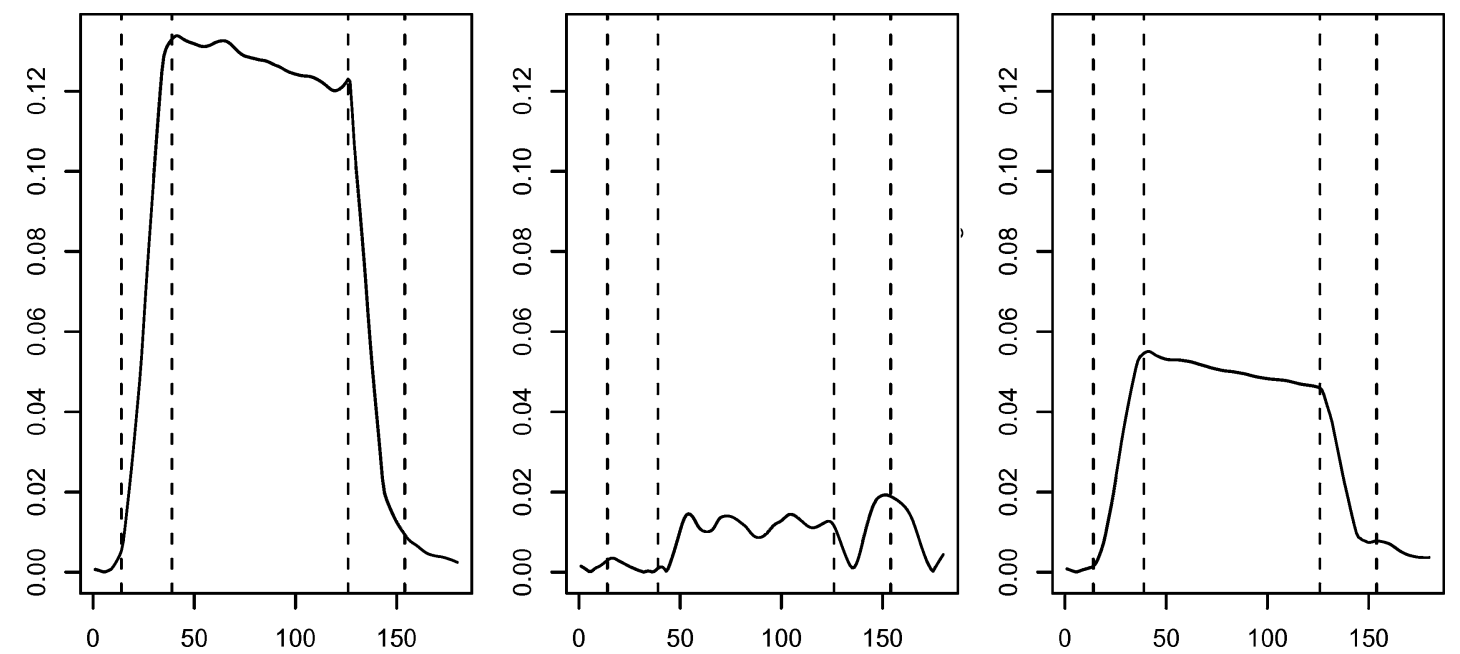

Fig. 2. Selected relative change from rest for a smile. Left: 13-17 (Upper Lip). Center: 4-5 (Eyebrow). Right: mean. Selected transitions shown 
transitions in the data. This was done anonymously to avoid bias in the selection. It would have been necessary to manually check the selections even had an automatic method been used so little time was lost and many errors avoided.

\subsection{B-Spline representation}

The curves shown in Fig. 2 can be approximated by standard cubic B-splines. We represent these curves as linear combinations of these basis functions, $B_{k}(t)$ for $k=1, \ldots, m$. The $l$ th curve, $r_{l}(t)$, where $l=1, \ldots, n(n-1) / 2$, is represented as

$$
r_{l}(t)=\sum_{k=1}^{m} c_{l k} B_{k}(t)+\varepsilon(t)
$$

where the coefficients $c_{l k}$ are found by minimizing a least squares criterion:

$$
\int_{0}^{1}\left(r_{l}(t)-\sum_{k=1}^{m} c_{l k} B_{k}(t)\right)^{2} d t
$$

Perhaps another more robust criterion could be used, but we chose to remove outliers and other aberrant points earlier in the process and so our earlier smoothing makes this unnecessary in this case. The particular B-spline basis is determined by the choice of knot location. We evenly space our knots within the five phases described above. Furthermore, we know that $r_{l}(0)=$ $r_{l}($ end $)=0$. We can impose this restriction directly by omitting the first and last B-spline basis functions. The B-spline basis functions corresponding to Fig. 2 with just one interior knot for each phase are shown in Fig. 3.

Because the transition points will differ from motion to motion, the placement of the knots will also differ. However, we will be able to directly compare and compute statistics on the coefficients $c$ with the assurance that $c_{l k_{1}}$ and $c_{l k_{2}}$ represent the same part of the motion. The knot positioning ensures the appropriate registration.

We chose $m=16$. This allows for 6 knots at the endpoints and transitions and two interior knots in the phases. Note that the choice of the number of knots is now an approximation rather than a smoothing issue. The observed data has already been smoothed (see Fig. 2). We simply need enough knots to

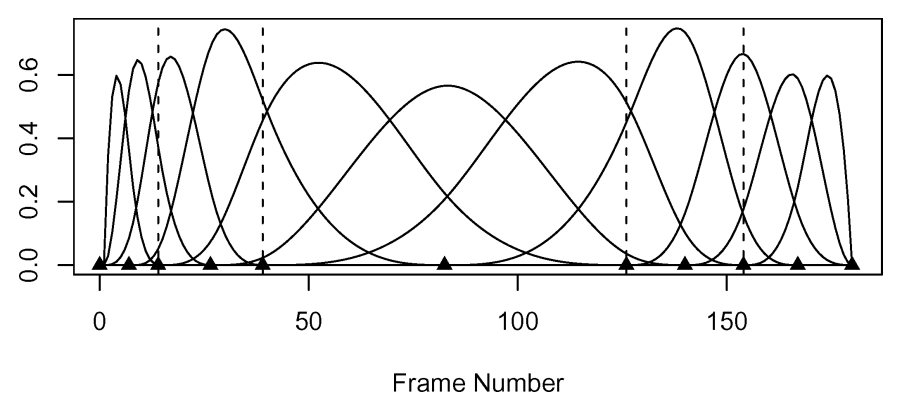

Fig. 3. B-spline basis functions corresponding to transitions in Fig. 2. Knot locations are shown on the horizontal axis. Note the zero values at the two endpoints adequately approximate the curves without using more than necessary which would increase the computational and storage burden.

\section{Statistics}

For convenience, we unroll the matrix $c_{l k}$ into a vector $c_{h}$ where $h=1, \ldots, \operatorname{mn}(n-1) / 2$ which represents one complete motion. For our choices of $m=16$ and $n=24$, we have a vector of length 4416. Also, we have the distance between markers at rest $d_{i j}(0)$ which can also be unrolled into a vector $d_{g}$ where $g=1, \ldots, n(n-1) / 2$ which represents the face at rest. What follows are standard statistical methods for such a multivariate responses, $c_{h}$ and $d_{g}$, with some special adaptations and interpretations for this particular use.

For given $c_{h}$ and $d_{g}$, we can generate a facial motion by converting $d_{g}$ back into the matrix $d_{i j}(0)$ and computing $r_{l}(t)$ from $c_{h}$ and so finding $r_{i j}(t)$. We can then form

$$
d_{i j}(t)=d_{i j}(0)\left(1+r_{i j}(t)\right)
$$

and so reconstruct the whole motion by the methods explained earlier.

Most of the intermarker pairs used have a symmetric counterpart on the other side of the face. We have chosen to symmetrize the facial shape and motion by averaging these pairs in the examples that follow. Note that such symmetrization will not always be appropriate.

\subsection{Means}

It is straightforward to compute means within different subgroups in the data. For example, we may compute the average smile on the average face by simply averaging $c_{h}$ and $d_{g}$ over all the observed smiles. To compute the resulting motion, we must also specify the 4 transitions. We could compute the means of the observed transitions but for ease of comparison between different displays, we have set these transitions at $t=1 / 6,2 / 6,4 / 6,5 / 6$. Such a smile is shown in Exhibit 2.

It is not necessary to use the same subgroups for computation of the motion and the static face. We could compute

$$
d_{i j}(t)=d_{i j}^{A}(0)\left(1+r_{i j}^{B}(t)\right)
$$

where $\mathrm{A}$ and $\mathrm{B}$ represent means computed over different groups of individuals or even just a single individual. For example, we can impose the group average smile on the face of any given individual. Within this particular application, this would be useful for comparing the actual motion of a patient with what might considered normal motion.

We can also decompose the effects of static shape and dynamic motion. We might ask whether a particular subgroup differs from another because the motion or the shape or both are different. To illustrate this idea consider a division of our subjects into two groups based on their MFLF score. One group consists of subjects considered normal and the other with higher scores 

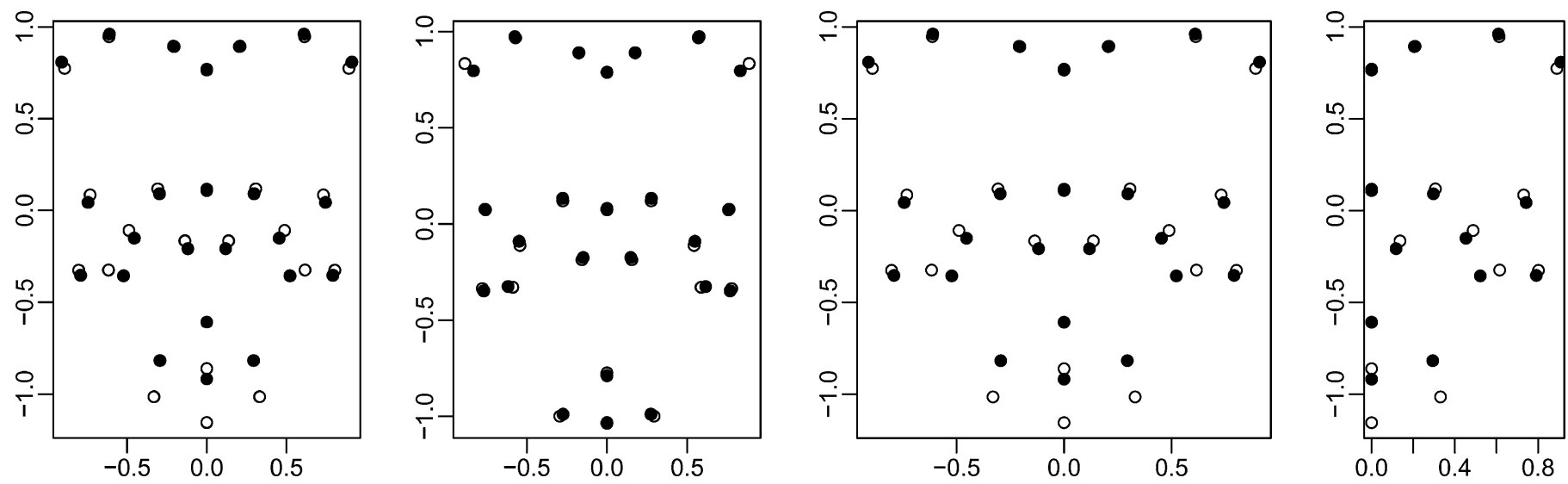

Fig. 4. In the first panel, the average normal face and motion is compared with the average patient face and motion. In the second panel, the average normal face and motion is compared with the patient motion applied to the average normal face. The midpoint of the motion is depicted. Markers of the normal subject is shown with hollow dots and patient with solid dots

generally tending to indicate smaller jaws. We call the higher MFLF group, "patient" for ease of reference.

In Exhibit 3, we show a comparison of average smiles of normal subjects on the average normal face with the average smile of the patient subjects on the average patient face. We observe some clear differences in these motions (ignoring questions of statistical significance for now). But is the difference because the patient group have different shaped faces or because they smile differently? In Exhibit 4, we show a comparison of average smiles of normal subjects on the average normal face with the average smile of the patient subjects on the average normal face. The differences we now see are just those of motion which are far less substantial. Although it is best to see these differences in a dynamic display, we show the frontal view in Exhibits 3 and 4 at the midpoint of the motion in Fig. 4.

This observation has some practical significance since surgical techniques exist for extending the jaws of retrognathic patients. One might be concerned that altering the shape of the face would not be sufficient if the motion were abnormal. However, our exhibits suggest that the differences are due to abnormalities in shape, not in motion and that provided the surgery did not alter the motion of the patient, the outcome would be normal shape and motion. However, since this conclusion is derived from just a single type of motion (a smile) and a single measure of facial shape (MFLF) it is just speculation. A broader analysis is under way.

\subsection{Variance}

Of course, there is substantial natural variation in facial motion. We can describe the nature of this variation with a principal components analysis on the $c_{h}$. In this case, we have 4416 variables but even counting all the smiles separately, we have only 145

Fig. 5. Front and side views of the smile at maximum expression. Average plus and minus two standard deviations in the direction of the first principal component are shown

cases. Nevertheless, we may still compute the principal components. We find the percentage of variation explained by the first 5 components are $32.6,12.9,4.6,4.4$ and $4.1 \%$ respectively. We compute

$$
\bar{c} \pm 2 \sqrt{s_{i}} v_{i}
$$

where $s_{i}$ and $v_{i}$ are the $i$ th eigenvalue and eigenvector respectively. We have applied these motions for the first principal component to the average face as shown in Exhibit 5 and Fig. 5. We see that the first principal component reflects the variation between people who tend to open their mouth when smiling as opposed to those who tend to keep their mouths closed.

The principal component scores are useful to identify unusual motions. These can be used to detect faulty or exceptional motions that were not found at an earlier stage. Furthermore, these can be used to rate new patients with respect to a standard group. This provides a quantitative measure of abnormality and could also be used to objectively assess any changes due to surgery.

\subsection{Inference}

We could simply apply the standard techniques of multivariate analysis (see for example, Johnson and Wichern 2002) to the $c_{h}$. However, the dimension is large (1056 in our example) and so the power of such tests would be poor in that they would reflect unimportant differences between the groups arising in the smaller principal components. A similar problem of dimensionality with functional data was observed in Faraway (1997). Instead, we contend that it is both better and simpler to perform the inference on the first few principal component scores.

In Fig. 6, we show the first three principal component scores plotted against MFLF. No relationship is apparent although some groupings of the three replicates per subject can be seen. We can fit a linear mixed effects model for the $j$ th replicate of 

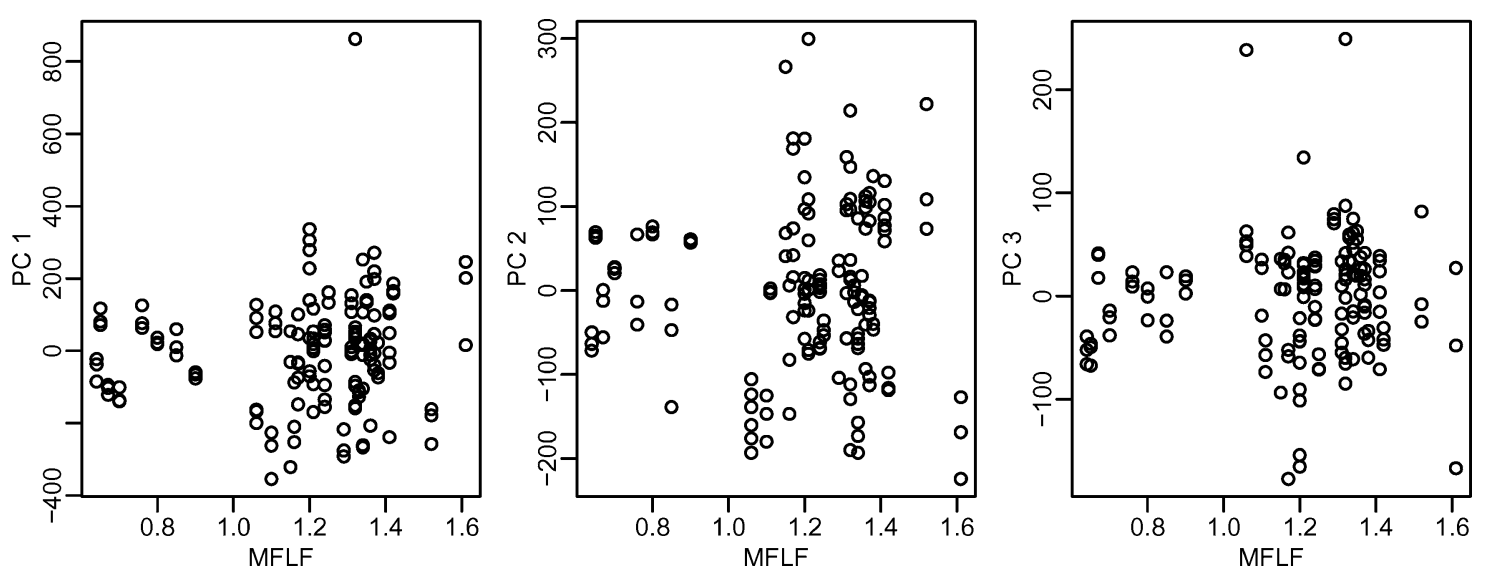

Fig. 6. First three principal components of the smile against MFLF

subject $i$ :

$$
p c_{i j}=\beta_{0}+\beta_{1} \mathrm{MFLF}_{i}+\gamma_{i}+\varepsilon_{i j}
$$

where $\gamma_{i}$ is the random subject effect with variance $\sigma_{\gamma}^{2}$ while within subject variation $\varepsilon_{i j}$ has variance $\sigma_{\varepsilon}^{2}$.

As might be expected, the test $H_{0}: \beta_{1}=0$ is not rejected $(p=0.45)$. This confirms our earlier observation about the small difference in the two motions. We find an intraclass correlation coefficient of $62.7 \%$. This indicates that while there is some consistency in smiles of the same subject, there is also a fair amount of variation in those smiles.

More extensive modeling and inference is possible but not explored here.

\section{Discussion}

For this particular application and other similar clinical problems, we have developed methods for

- Comparing and testing the dynamic motion of groups of subjects independent of their static facial shape.

- Describing and visualizing variation within the facial motions of groups of subjects

- Scoring the difference between individual subjects and the population motion

We also believe the analysis presented here has application to fields beyond facial modeling in clinical applications. Any data on continuously changing shapes might be modeled in a similar manner.

\section{Appendix: Motion viewer}

Facial motions are difficult to represent statically and in two dimensions so they are best viewed dynamically. We have constructed a viewer that can display these motions that can be view at any angle. The viewer may be downloaded from

$$
\text { http://www.stat.lsa.umich.edu/ }{ }^{\text {faraway/face/ }}
$$

The viewer keyboard commands are

- Function keys F1-F5-Load Exhibit 1-5 respectively

- Arrow keys rotate the view

- a-show first (or only) face moving

- $\mathrm{b}$ - show second (if available) face moving

- $\mathrm{c}$ - show both (if available) faces moving

- $</>$-increase/decrease face size

List of exhibits

1. Two smiles by the same individual.

2. The average smile.

3. Average face/smile of normal MFLF group compared with average face/smile of patient MFLF group.

4. Average face/smile of normal MFLF group compared with average face of normal with average smile of the patient MFLF group.

5. Smiles two standard deviations above and below the average in the direction of the first principal component.

\section{Acknowledgments}

Dr. Carroll-Ann Trotman of University of North Carolina Dental School collected the data and motivated the analysis discussed in this article. This work was supported in part by grant DE 05215-2151 from the National Institute of Dental Research.

\section{References}

Bookstein F. 1991. Morphometric Tools for Landmark Data: Geometry and Biology. Cambridge University Press.

Dryden I. and Mardia K. 1998. Statistical Shape Analysis. Wiley.

Faraway J. 1997. Regression analysis for a functional response. Technometrics 39: 254-261.

Johnson R. and Wichern D. 2002. Applied Mulivariate Statistical Analysis, 5th edition. Prentice Hall, New Jersey. 
Lele S. and Richtsmeier J. 2000. An Invariant Approach to Statistical Analysis of Shapes. Chapman \& Hall.

Mendez M. 1999. A three-dimensional analysis of facial movement in normal adults: Methodological variation and characterization of natural expressions. Master's thesis, University of Michigan.

Ramsay J. and Li X. 1998. Curve registration. Journal of the Royal
Statistical Society, Series B 60: 351-363.

Small C. 1996. The Statistical Theory of Shape. Springer.

Trotman C.-A., Faraway J., and Essick G. 2000. Three-dimensional nasolabial displacement in repaired unilateral and bilateral cleft lip and palate patients. Plastic and Reconstructive Surgery 105(4): $1273-1283$. 